\title{
Preparation and characterization of brushite crystals using high internal phase emulsion.
}

\begin{abstract}
Various morphologies of brushite crystals were successfully synthesized using oil-in-water high internal phase emulsion stabilized by surfactants with various polyoxyethylene chain lengths for the first time. Rheological measurements had proven that such emulsion was a highly stable reaction media for the synthesis of particulate and porous brushite crystals. Rheological properties of the emulsion with and without the presence of crystal growth were compared and discussed. Morphologies of brushite crystals comprised of leafletlike particles to continuous dendritic network structure which gave rise to porosity in submicron size with increasing polyoxyethylene chain length. The mechanism leading to the various types of morphologies produced is discussed schematically based on small angle X-ray scattering results.
\end{abstract}

Keyword: Brushite; High internal phase emulsion; Porous. 